\title{
MEDOC 坐标系及其对测定极移的影响*
}

\author{
朱文耀 \\ A. Puizzi \\ 丁元君 \\ （中国科学院上海天文台） \\ (GRGS/CNES, France) \\ （中国科学院紫金山天文台,南京）
}

目前全球同时存在两个卫星多普勒测定极移的永久网. 一个是美国国防制图局负责的 DMA 网; 另一个是法国大地测量研究所负责的 MEDOC 网. 这两个全球网作为日常工作分别 给出 1 至 2 颗子午卫星的精密星历和测得的极位置. 虽然目前 MEDOC 精密星历和测得的 极位置的精度还比不上 DMA。 但由于 MEDOC 是一个纯粹科研性质的网, 观测资料和精密 星历均向外公开，在卫星多普勒测地工作中具有较大的实用价值. 特别是中国已有两个台站 (上海天文台和紫金山天文台)参加了 MEDOC 网,资料交换有了正常的途径. MEDOC 网收 集的观测资料和 MEDOC 精密星历将在中国的卫星多普勒测地工作中发挥较大的作用. 为 准确地应用 MEDOC 网的资料, 也为了对 DMA 和 MEDOC 测定极移的结果进行比对, 有必 要对以下的问题进行深入的研究: (1) MEDOC 坐标系的确切定义; (2) MEDOC 坐标系 与其他空间大地测量坐标系的关系; (3) MEDOC 坐标系对测定极位置的影响.

\section{一、MEDOC 坐 标 系}

MEDOC 试验开始于 1977 年, 有 11 个台站,其中 7 个与 DMA 网相同. 这 11 个台站的 坐标是通过 14 个六天周期用半动力法独立解算求得. 解的标准偏差约为 3 米 ${ }^{[1]}$. 随后参加 的台站的坐标是在固定这些老站坐标的基础上, 用同样的半动力法求得, 计算中采用 GEM-10 地球引力场模型.

被称为 JEU-SEPT78 的 MEDOC-1 坐标系, 并不依附于 DMA 的 NSWC-9Z-2 坐标 系, 它是一个独立的坐标系. 应该说这个坐标系的坐标原点、经度零点和 $Z$ 轴的指向是由这些 站坐标的坐标零点、经度零点和它们的纬度值所体现的极的平均值来定义的.

由于 DMA 网的站坐标向外保密, JEU-SEPT78 与 NSWC-9Z-2 之间的转换关系一直未 能研究确定. 致使 JEU-SEPT78 坐标系与别的基于全球资料测定的空间大地测量坐标系(如 SLR、VLBI 等坐标系)之间的转换关系均未一一建立. 由此限制了 MEDOC 精密星历在测 地工作中的广泛应用.

为了建立 JEU-SEPT78 与 NSWC-9Z-2 坐标系之间的转换关系, 作者通过直接和间接 的各种途径收集和计算了九个台站在该两个坐标系内的三维直角坐标,具体情况如下:

1. Mizusawa (Japan), Smithfield (Astralie) 和 Hernden (USA) 三站分别属于 DMA 和 MEDOC 网. 在 MEDOC 建网开始时由 DMA 提供了该三站在 NWL-9D 坐标系内的三维 地心直角坐标. 我们利用文献. [2] 发表的 NWL-9D 与 NSWC-9Z-2 之间的转换参数将它们 转换成 NSWC-9Z-2 坐标系内的直角坐标. Mizusawa 站的站坐标直接取自文献 [3].

本文 1985 年 5 月 23 日收到.

* 本文是作者在法国空间研究中心,空间大地测量研究所 (GRGS/CNES) 工作期间完成的. 
2. Uccle (Belgique) 和 Ottawa (Canada) 站同属 DMA 和 MEDOC 网. 在文献 [4] 中 P. Paqnet 等根据该站九年的观测资料分别以 ORB 和 DMA 的 DARCUS 两个软件用 DMA 的精密星历计算了 Uccle 站在 NSWC-9Z-2 坐标系的 $(\lambda, \varphi, H)$. 我们取其平均值. Ottawa 站的 $(\lambda,-\varphi, H)$ 取自文献 (5). 然后根据 NWL-10E-1 地球引力场模型参数将两站的球坐 标化为三维直角坐标. Wettzell (Germary) 站仅属于 MEDOC 网. 文献 [6]作者 Sehüter 利用 DMA 精密星历由该站 1978.3-1981.7 的观测资料以单点定位的方法测定了该站在 NSWC9Z-2 坐标系中的三维直角坐标平均值.

3. Graz (Austria) 站仅属于 MEDOC 网. 据文献 [7] 的联合解 2, 该站在 NSWC-9Z-2 中坐标值是用该联合解中 Graz 和 Wettzell 站间的相对坐标通过 Wettzell 站的坐标值求得.

4. Shangai (China) 站仅属于 MEDOC 网. 该站在 MERIT 主联测期间与相距不远的 激光测卫站进行了并址（colocation）观测，两站的相对位置通过地面大地测量的方法精确测 定. 激光站在 SLR 坐标系内的点位由美国 Texas 大学精确测定 (在该坐标系内 STAIAS 激 光站的经度为 $\left.283^{\circ} 10^{\prime} 19^{\prime \prime} 945\right)$. 由此我们可以归算出多普勒观测站在 SLR 坐标系内的点位, 然后再根据 SLR 坐标系与 NSWC-9Z-2 系的转换参数 ${ }^{[8]}: \Delta Z=-4 m, K=0.5 \mathrm{ppm}, Q_{z}=$ - .78", 求得该站在 NSWC-9Z-2 坐标系内的三维直角坐标.

5. Grasse (France) 站,既不属于 DMA 网又不属于 MEDOC 网。但它提供了一个外部 符合的检验。该站在 NSWC-9Z-2 系统内的坐标由 Saint-Crit 利用 DMA 精密星历精确测 定. 在文献 [9] 中, Saint-Crit 等基于同样的卫星, 同样的观测周期以及同样的计算软件利用 MEDOC 精密星历计算了 Uccle 和 Grasse 站的坐标. 我们用其相对点位, 通过 Uccle 站求 得该站在 JEU-SEPT78 坐标系内的坐标 .

通过以上途径所求得的这九个台站在 NSWC-9Z-2 和 JEU-SEPT78 坐标系内的坐标由 表 1 列出.

表 1 Wettzel1 等九个台站在 NSWC-9Z-2 和 JEU-SEPT78 系内的直角坐标

\begin{tabular}{|c|c|c|c|c|c|c|}
\hline \multirow{2}{*}{ 台 站 } & \multicolumn{3}{|c|}{ NSWC-9Z-2 } & \multicolumn{3}{|c|}{ JEU-SEPT 78} \\
\hline & $x$ & $\gamma^{\prime}$ & $Z$ & $\lambda$ & $y$ & $z$ \\
\hline Wettzell & 4075541.0 & 931805.2 & 4801606.9 & 4075545.6 & 931796.2 & 4801600.1 \\
\hline Graz & 4194432.8 & 1162684.9 & 4647243.8 & 4194436.0 & 1162675.7 & 4647233.1 \\
\hline Uccle & 4027837.3 & 307000.0 & 4919535.0 & 4027839.4 & 306989.1 & 4919528.0 \\
\hline Grasse & 4081937.9 & 556365.8 & 4389075.6 & 4081940.0 & 555356.0 & 4389069.9 \\
\hline Shangai & -2830934.3 & 4676467.2 & 3275113.3 & -2830932.3 & 4676477.0 & 3275106.7 \\
\hline Mizusawa & -3857185.0 & 3108678.1 & 4004045.2 & -3857182.0 & 3108689.5 & 4004040.7 \\
\hline Smithtield & -3942229.0 & 3468874.3 & -3806202.6 & -3942222.5 & 3468886.9 & -3608214.5 \\
\hline Hernden & 1090123.8 & -4842534.3 & 3991971.9 & 1090113.7 & -4842537.4 & 3991966.4 \\
\hline Ottawa & 1091435.7 & -4351296.8 & 4518696.7 & 1091425.9 & -4351301.4 & 4518692.8 \\
\hline
\end{tabular}

采用如下七个的坐标转换公式:

$$
\left(\begin{array}{l}
X \\
\mathbf{Y} \\
Z
\end{array}\right)_{\mathrm{DMA}}=\left(\begin{array}{l}
\Delta X \\
\Delta Y \\
\Delta Z
\end{array}\right)+(1+K)\left(\begin{array}{rrr}
1 & -Q_{z} & Q_{y} \\
Q_{z} & 1 & -Q_{x} \\
-Q_{y} & Q_{x} & 1
\end{array}\right)\left(\begin{array}{l}
X \\
\mathbf{Y} \\
Z
\end{array}\right)_{\text {MEDOC }} .
$$


以下列 4 种方案通过最小二乘平差所求得的七个转换参数及其标准偏差由表 2 列出.

方案 1, 由 Uccle, Mizusawa, Smithfield, Hernden 和 Ottawa 等五个站进行转换平差, 为 使测站分布保持全球平衡.

方案 2, 除去 Grasse, Shangai 和 Ottawa 站其余的站进行转换平差, 为研究在某些测站 不观测时 MEDOC 系的稳定性.

方案 3, 除了 Grasse 和 Shangai 两站, 其余的站均参加转换平差. 因为这两个测站提供 了一个外符检验.

方案 4, 九个测站均参加转换平差.

表 2 DMA 和 MEDOC-1 坐标系间的七个转换参数

\begin{tabular}{c|c|c|c|c|c|c}
\hline$\Delta X(\mathrm{~m})$ & $\Delta Y(\mathrm{~m})$ & $\Delta Z(\mathrm{~m})$ & $K(\mathrm{ppm})$ & $Q_{X}$ & $Q_{Y}$ & $Q_{Z}$ \\
\hline (1) $.22 \pm .45$ & $-.52 \pm .45$ & $8.27 \pm .45$ & $-.616 \pm .070$ & $.007^{\prime \prime} \pm .017$ & $.005^{\prime \prime} \pm .018$ & $.492^{\prime \prime} \pm .019$ \\
$(2) 1.08 \pm .41$ & $-.47 \pm .41$ & $9.14 \pm .41$ & $-.494 \pm .064$ & $.007^{\prime \prime} \pm .016$ & $-.026^{\prime \prime} \pm .015$ & $.503^{\prime \prime} \pm .018$ \\
(3) $.42 \pm .38$ & $.00 \pm .38$ & $8.92 \pm .38$ & $-.453 \pm .059$ & $.045^{\prime \prime} \pm .015$ & $.006^{\prime \prime} \pm .014$ & $.518^{\prime \prime} \pm .017$ \\
(4) $1.30 \pm .33$ & $-.21 \pm .33$ & $8.66 \pm .33$ & $-.561 \pm .052$ & $.005^{\prime \prime} \pm .013$ & $-.020^{\prime \prime} \pm .012$ & $.471^{\prime \prime} \pm .015$ \\
\hline
\end{tabular}

根据上述的四个方案加上欧洲四个测站的转换平差,所求得的三参数 $\left(\Delta Z, K, Q_{s}\right)$ 的转 换解由表 3 列出.

表 3 三参数的转换解

\begin{tabular}{c|c|c|c|c|c}
\hline$\Delta Z$ & $8.24 \pm .45$ & $9.25 \pm .41$ & $8.77 \pm .38$ & $8.65 \pm .33$ & $8.69 \pm .50$ \\
$K$ & $-.610 \pm .070$ & $-.486 \pm .064$ & $-.418 \pm .059$ & $-.529 \pm .052$ & $-.244 \pm .079$ \\
$Q_{Z}$ & $.49^{\prime \prime} \pm .02$ & $.49^{\prime \prime} \pm .02$ & $.49^{\prime \prime} \pm .02$ & $.46^{\prime \prime} \pm .02$ & $.48^{\prime \prime} \pm .02$ \\
\hline
\end{tabular}

表 4 由表 $2 、 3$ 的转换参数所求得的站坐标的偏差(以米为单位)

\begin{tabular}{|c|c|c|c|c|c|c|c|c|c|c|c|c|c|c|c|}
\hline \multirow{2}{*}{ 台 站 } & \multicolumn{3}{|c|}{1} & \multicolumn{3}{|c|}{2} & \multicolumn{3}{|c|}{3} & \multicolumn{3}{|c|}{4} & \multicolumn{3}{|c|}{5} \\
\hline & $X$ & $Y$ & $z$ & $X$ & $Y$ & $Z$ & $x$ & $Y$ & $Z$ & $X$ & $Y$ & $Z$ & $X$ & $Y$ & $Z$ \\
\hline Ottawa & 0.2 & 0.0 & 1.4 & & & & 0.9 & -0.9 & 2.0 & 0.6 & 0.0 & 2.2 & & & \\
\hline Wettzell & & & & -0.2 & -0.1 & 0.5 & -0.3 & -0.3 & 0.3 & 0.2 & -0.5 & -0.4 & 0.4 & 0.3 & 0.7 \\
\hline Graz & & & & -1.2 & -0.2 & -3.3 & -0.1 & -0.2 & -3.5 & -0.8 & -0.6 & -4.2 & -.5 & 0.3 & -3.1 \\
\hline Uccle & -0.8 & -2.2 & 1.8 & -0.2 & -1.9 & 0.2 & -0.2 & -2.0 & -0.1 & 0.2 & -2.2 & -0.7 & 0.4 & -1.6 & 0.5 \\
\hline Grasse & & & & & & & & & & -0.7 & 0.0 & 1.0 & -.3 & 0.8 & 1.9 \\
\hline Shangaj & & & & & & & & & & 3.2 & -0.6 & 1.2 & & & \\
\hline Mizusawa & -1.3 & -0.4 & 1.5 & -1.7 & -0.1 & 2.3 & -1.1 & 0.5 & 1.5 & -0.4 & 0.5 & 1.6 & & & \\
\hline Smithfield & 0.6 & 1.0 & -1.5 & 1.5 & 0.9 & -1.4 & 0.1 & 1.9 & -0.7 & 2.6 & 1.5 & -1.5 & & & \\
\hline Hernden & 0.8 & 2.5 & 0.3 & 1.8 & 1.4 & 1.6 & 1.9 & 1.0 & 0.6 & 1.5 & 1.8 & 0.9 & & & \\
\hline
\end{tabular}

由表 2 的 4 组和表 3 的第 5 组转换参数, 所求得的各站坐标的偏差由表 4 给出.

从表 $2 、 3$ 和表 4 可看出, 七个转换参数中 $\Delta Z, K, Q_{z}$ 三个参数基本上是可靠的. $\Delta X$ 有一 个正向的偏差相对而言也有一定的可靠性. 其余三个参数本身数值较小与其标准偏差属同一 
量级, 无多大意义. 故总的来说 MDA 和 MEDOC-1 之间的转换关系可用 $\Delta Z \doteq 9 \mathrm{~m}, K \doteq$ $-0.5 \mathrm{ppm}, Q_{z}=0$. . 5 来表述.

MEDOC-1 坐标系与其他全球性的空间大地测量坐标系(如 SLR, VLBI 坐标系) 比较可 得如下的关系 ${ }^{[8]}$ : (1) MEDOC-1 坐标系存在一个大约 $1 \mathrm{ppm}$ 的尺度 偏差; (2) 与 SLR 坐标 系相比较 MEDOC-1 坐标系的 $Z$ 分量存在约 12-13 米的偏差; (3) MEDOC-1 坐标系的经 度(东)需增加 1. '. 3 才能使它的零子午面与 VLBI 系的零子午面重合.

从表 4 可看出 MEDOC-1 坐标系的测站坐标确实存在 2-3 米的误差. 特别是 Graz 站 的 $\mathrm{z}$ 坐标明显偏高 3-4 米. 这也可从与欧洲地区的几次多普勒会战的结果比较中看出 ${ }^{[7]}$.

值得注意的是从表 1 和表 3 中可看出不同的局部地区显示出不同的转换参数. 特别是反 映在尺度因子上，西欧地区（Wettzell 等四站）显然有一个比东亚地区（Mizusawa 和 Shangai 站)小得多的尺度因子. 其原因可能是由于在测定站坐标的半动力法所采用的地球引力场模 型 GEM-10 对不同地区有不同的适合程度引起的.

自 1984 年起, MEDOC 在处理 MERIT 主联测的多普勒资料时对地球引力场等力学模 型作了一定的调整, 并以近一个月的观测资料用二天周期的半动力法重新计算各测站的地心 坐标. 解算中某些站的坐标保持固定. 解的标准偏差为 1 米, 由此建立了 MEDOC-2 网. 从 MEDOC-1 到 MEDOC-2 许多测站作了迁移, 我们经过各种途径收集和计算了 New Mexico 等测站在 NSWC-9Z-2 系内的坐标. 表 5 列出了它们在两个坐标系内的直角坐标.

表 5 New Mexico 等八测站在 DMA 和 MEDOC-2 坐标系内的直角坐标

\begin{tabular}{l|r|r|r|r|r|r}
\hline \multirow{2}{*}{ 台 站 } & \multicolumn{3}{|c|}{ DMA } & \multicolumn{4}{|c}{ MEDOC-2 } \\
\cline { 2 - 7 } & \multicolumn{1}{|c|}{$X$} & \multicolumn{1}{|c|}{$Y$} & \multicolumn{1}{|c}{$Z$} & \multicolumn{1}{|c}{$X$} & \multicolumn{1}{|c}{$Y$} \\
\hline New Mexico & -1556231.4 & -5169447.4 & 3387236.6 & -1556206.9 & -5169449.9 & 3387241.2 \\
Anchorage & -2656171.9 & -1544365.4 & 5570652.1 & -2656166.1 & -1544377.9 & 5570649.6 \\
Samoa & -6100059.0 & -997168.7 & -1568317.9 & -6100058.4 & -997197.9 & -1568316.5 \\
Mizusawa & -3857186.8 & 3108678.7 & 4004047.4 & -3857198.0 & 3108662.0 & 4004045.7 \\
Uccle & 4027871.6 & 307012.2 & 4919512.2 & 4027872.0 & 307030.2 & 4919516.2 \\
Papeete & -5245227.2 & -3080463.7 & -1912840.2 & -5245215.5 & -3080484.1 & -1912840.7 \\
Hernden & 1090121.9 & -4842531.6 & 3991976.1 & 1090121.9 & -4842523.8 & 3991978.0 \\
Shangai & -2830932.0 & 4676471.1 & 3275114.2 & -2830952.5 & 4676458.1 & 3275117.2 \\
\hline
\end{tabular}

由上述坐标转换公式求得的两坐标系之间的七转换参数和三参数的解由表 6 列出. 用七 个转换参数计算的各测站坐标的偏差由表 7 给出. 从表 6 可看出 MEDOC-2 坐标系的经度相 对于 DMA 增加了 $0 .{ }^{\prime} 9$, MEDOC-2 的坐标原点沿 $z$ 轴上升了 1.3 米. 其他几个转换参数意 义不大. 总的说来 MEDOC-2 坐标系已比较接近 BIH 坐标系统. 从表 7 可看出 MEDOC-2

表 6 DMA 和 MEDOC-2 坐标系间的转换参数

\begin{tabular}{|c|c|c|c|c|c|c|}
\hline$\Delta X(\mathrm{~m})$ & $\Delta Y(\mathrm{~m})$ & $\Delta Z(\mathrm{~m})$ & $K(\mathrm{ppm})$ & $Q_{\mathrm{X}}$ & $Q_{Y}$ & $Q_{z}$ \\
\hline $.17 \pm .35$ & $-.60 \pm .35$ & $\begin{array}{l}-1.29 \pm .35 \\
-1.37 \pm .35\end{array}$ & $\begin{array}{l}.029 \pm .055 \\
.039 \pm .055\end{array}$ & $018^{\prime \prime} \pm .014$ & $.303^{\prime \prime} \pm .014$ & {$\left[\begin{array}{l}-.905^{\prime \prime} \pm .014 \\
-.890^{\prime \prime} \pm .014\end{array}\right.$} \\
\hline
\end{tabular}


表 7 由表 6 的七参数求得的站坐标的偏差

\begin{tabular}{c|c|c|c|c|c|c|c|c}
\hline & New Mexico & Anchorage & Samoa & Mizusaw a & Uccle & Papecte & Hernden & Shangai \\
\hline$\Delta X(\mathrm{~m})$ & 2.0 & -.8 & -3.8 & 2.6 & 2.1 & -1.8 & -.4 & .2 \\
$\Delta Y(\mathrm{~m})$ & 3.3 & -2.0 & -2.8 & -.5 & -.7 & 2.1 & 1.9 & -1.3 \\
$\Delta Z(\mathrm{~m})$ & 3.0 & -3.7 & -.3 & -2.6 & 2.8 & -2.0 & .3 & 2.2 \\
\hline
\end{tabular}

的站坐标存在 3-4 米的误差,这也可从利用 MEDOC-2 精密星历单点定位的结果看出.

\section{二、MEDOC 坐标系对测定极位置的影响}

$M E D O C$ 坐标系存在较大的尺度因子和 $\Delta Z$ 的偏差对测站的纬度和高程的影响可用下式 计算:

$$
\Delta \phi^{\prime \prime}=\Delta Z /[30.88 / \cos \phi-0.21 \cos \phi], \Delta H=6370000 K+\Delta Z \sin \phi .
$$

根据 Cardine 的研究表明 ${ }^{[10]}$ : 经度零点和尺度因子的偏差并不会对极位置的测定产生影 响. 同时从 (2) 式也可看出 $\Delta Z$ 的偏差也不可能对极位置产生有意义的影响. 故 MEDOC 网 虽存在较大的经度、尺度和 $\Delta Z$ 的系统偏差，它对极位置的测定并不会产生实质性的影响.

值得注意的是坐标系的稳定性. 如前所述 MEDOC 坐标系的坐标原点,经度零点等是由 MEDOC 测站的坐标零点平均值, 经度零点平均值来定义的. 由于 MEDOC 测站的站坐标存 在一定的误差，参加的台站有限；而不同的时期提供资料的测站不尽相同，每个测站所观测 到的卫星通过数也不一样. 这就导致在不同的时期，由不同的测站，不同的资料分布所组 成的 MEDOC 坐标系是不同的. 表 2 的四组转换参数的差别正说明了这一点. 故严格地说 MEDOC 坐标系是不稳定的. 作者认为这种不稳定性同样存在于其他空间测地工作中. 站坐 标的误差越大参加的测站越少, 这种坐标系不稳定的程度就越大. 对 MEDOC 来说, 由于站 坐标的误差较大，这种不稳定性已大到足以影响测定极位置的结果. MEDOC 的极位置与 DMA、BIH 的极位置相比, 在不同时期出现相对而言不同的系统偏差, 其原因之一就是由于 不同时期 MEDOC 网由不同台站组成所引起的. 解决坐标系不稳定性的途径: 一是不断提 高台站的坐标精度; 二是增加全球分布的台站个数; 三是尽量保证参加测站的相对稳定.

\section{参 考 文 献}

[ 1 ] Gambis, D. et a!., Bull. Geod., 54(1980), 103-118.

[ 2 ] Muneendra Kumar, 3D. 1982*, 169-178.

[ 3 ] Shigeru Yumi, 2D. 1979, 767-782.

[ 4 ] Paquet, P. et al., 3D. 1982, 539-556.

[ 5] Ouba, J. Annales de Geophysique, 37(1981), 1: 205-212.

[ 6 ] Schluter, W. et al., 3D. 1982, 575-587.

[ 7 ] Ehlert, D. et al., 3D. 1982, 447-462.

[ 8 ] Hothem, L. D. et al., 3D. 1982, 109-128.

[ 9 ] Saint-Crit, L., Manuscripta Geodetica, 6(1980), 335-353.

[10] Caroline, F. L., 3D. 1982, 129-150.

* 3D. 1982: Proceedings of 3rd International Geodetic Symposium on Satellite Doppler Positionind. Feb. 1982. 